\title{
Validating the FLASH Code: Vortex-Dominated Flows
}

\author{
Greg Weirs, Vikram Dwarkadas, Tomek Plewa \\ ASCI FLASH Center, University of Chicago vikram@flash.uchicago.edu, \\ tomek@flash.uchicago.edu, weirs@flash.uchicago.edu \\ Chris Tomkins and Mark Marr-Lyon \\ Los Alamos National Laboratory ctomkins@lanl.gov, mmarr@lanl.gov
}

\begin{abstract}
As a component of the Flash Center's validation program, we compare FLASH simulation results with experimental results from Los Alamos National Laboratory. The flow of interest involves the lateral interaction between a planar $\mathrm{Ma}=1.2$ shock wave with a cylinder of gaseous sulfur hexafluoride $\left(\mathrm{SF}_{6}\right)$ in air, and in particular the development of primary and secondary instabilities after the passage of the shock. While the overall evolution of the flow is comparable in the simulations and experiments, small-scale features are difficult to match. We focus on the sensitivity of numerical results to simulation parameters.
\end{abstract}

\section{Introduction}

The impulsive acceleration of a material interface can lead to complex fluid motions due to the Richtmyer-Meshkov (RM) instability. Here, the misalignment of pressure and density gradients deposits vorticity along the interface, which drives the flow and distorts the interface. At later times the flow may be receptive to secondary instabilities, most prominently the Kelvin-Helmholtz instability, which further increase the flow complexity and may trigger transition to turbulence.

Verification and validation are critical in the development of any simulation code, without which one can have little confidence that the code's results are meaningful. FLASH is a multi-species, multidimensional, parallel, adaptive-mesh-refinement, fluid dynamics code (C) 2018 Kluwer Academic Publishers. Printed in the Netherlands. 
for applications in astrophysics [1]. Calder et al. discuss initial validation tests of the FLASH code [2]. Herein we continue our validation effort by comparing FLASH simulations to an RM experiment performed at Los Alamos National Laboratory 3, 4,

\section{Experimental Facility and Data}

The experimental apparatus is a shock-tube with a $7.5 \mathrm{~cm}$ square crosssection, as shown in Fig. 1. Gaseous $\mathrm{SF}_{6}$ flows from an $8 \mathrm{~mm}$ diameter nozzle in the top wall of the shock-tube, forming a cylinder of dense gas in the otherwise air-filled test section. $\mathrm{A} \mathrm{Ma}=1.2$ shockwave travels through the shock-tube and passes through the cylinder. Our interest is in the resulting evolution of the $\mathrm{SF}_{6}$. All the experimental data is obtained in a plane normal to the cylinder axis, $2 \mathrm{~cm}$ below the top wall of the test section. The experiment is nominally two-dimensional; however, air diffuses into the $\mathrm{SF}_{6}$ column as it flows downward, thickening the interface and reducing the peak concentration of the heavy gas.

The initial $\mathrm{SF}_{6}$ distribution (before the shock impact) is visualized directly by Rayleigh-scattering from the $\mathrm{SF}_{6}$ molecules [3]. The pixel intensity in the experimental image gives only the mole fraction of $\mathrm{SF}_{6}$ relative to the peak mole fraction, $\mathrm{X}_{S F 6}$, which must be assumed. The distribution of $\mathrm{SF}_{6}$ is only approximately radially symmetric, and the signal is dominated by noise at the level of about $5-10 \%$. Smooth 

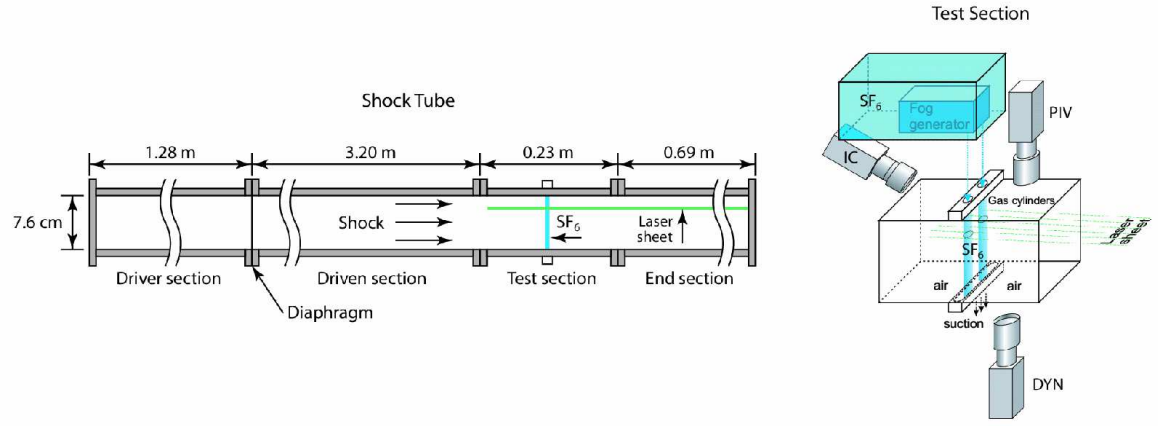

Figure 1. LANL shock tube dimensions with test section detail.

initial conditions for our simulations are obtained by fitting a radiallysymmetric function to the experimental data.

During the experiment the $\mathrm{SF}_{6}$ distribution is indirectly visualized by visible light scattering off water/glycol droplets, which are seeded in the $\mathrm{SF}_{6}$. A sequence of experimental images is shown in Fig. 2 The shock traverses the cylinder in less than $25 \mu \mathrm{s}$. The vortex Reynolds number of the flow, as measured experimentally, is $\operatorname{Re}=\Gamma / \nu \approx 5 \times 10^{4}$, where $\Gamma$ is the circulation and $\nu$ is the kinematic viscosity. Each image is taken from a different experimental run. The water/glycol droplets can also be used to construct two-dimensional velocity vectors in the image plane using particle image velocimetry (PIV) [5], but the entire test section must be seeded, so simultaneous velocity and composition measurements cannot be obtained. 


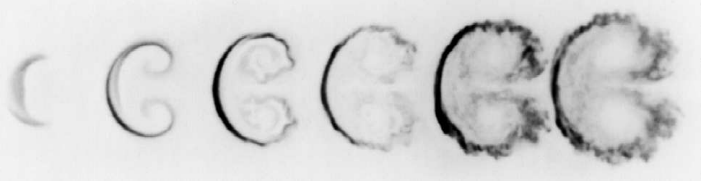

Figure 2. Experimental time series of the distribution of $\mathrm{SF}_{6}$. The first image corresponds to $50 \mu$ s after shock impact; following images are at 190, 330, 470, 610, and $750 \mu \mathrm{s}$. Intensity corresponds to the mole fraction of $\mathrm{SF}_{6}$.

\section{Flowfield Evolution}

As the shock traverses the cylinder, vorticity is deposited along the interface due to the misalignment of the pressure gradient (normal to the shock) and the density gradient (normal to the interface.) The density gradient arises from the gas composition; $\mathrm{SF}_{6}$ is about five times as dense as air. Once the shock has passed through the $\mathrm{SF}_{6}$, the flow is dominated by a counter-rotating vortex pair, as shown in Fig. 2. Instabilities develop along the distorted interface at the edge of the primary vortices. The development and evolution of the vortex pair and subsequent instabilities at the interface proceed in a weakly compressible regime. More precise descriptions can be found in the references [6, 7, 4].

The flowfield evolution is driven by flow instabilities and vortex dynamics, which are sensitive to the initial conditions and noise in the system. For validation this sensitivity is desireable because it provides a severe test for the FLASH code. Figure 3 shows a sequence of images from our baseline simulation. The minimum grid resolution is 78 microns, the initial peak mole fraction of $\mathrm{SF}_{6}$ is 0.6 , and the Courant 


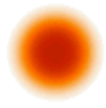

0

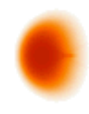

50

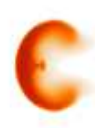

190

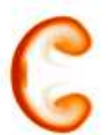

330

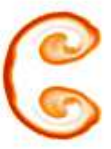

470

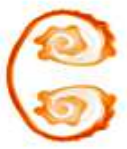

610

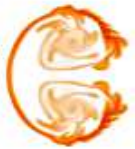

750

Figure 3. Evolution of the $\mathrm{SF}_{6}$, with time elapsed after shock impact listed in $\mu$ s. The mass fraction of $\mathrm{SF}_{6}$ is shown from a simulation in which $\mathrm{X}_{S F 6}=0.6$ and $\mathrm{CFL}=0.8$.

(CFL) number is 0.8. Overall the flow features in the simulation results are similar to those in the experimentally obtained images. Next we describe the effects of several simulation parameters on the computed results. The amount and location of small-scale structure, relative to the experimental data at $750 \mu \mathrm{s}$, will be used as a qualitative metric.

\section{Results}

In our initial investigations we have focused on the sensitivity of the computed solutions to several simulation parameters. We have considered the dependence on the initial maximum mole fraction of $\mathrm{SF}_{6}$, the mesh resolution, the mesh refinement pattern, and the Courant number. We have also compared velocity data, and are beginning to consider three-dimensional effects. Here we show results only for different mesh refinement patterns and Courant numbers. More thorough discussion of the results can be found in [8].

Regarding the initial maximum mole fraction of $\mathrm{SF}_{6}$, we find that simulations where $\mathrm{X}_{S F 6}=0.6$ seem to match the experimental results better than when $\mathrm{X}_{S F 6}=0.8$. At the higher value, the initial density gra- 
dient is larger; this leads to greater vorticity deposition, faster instability growth, and consequently, excessive small scale structure. However, the time sequences match better for $\mathrm{X}_{S F 6}=0.8$.

It is known that unavoidable discretization errors at discontinuous jumps in grid resolution can act as sources of spurious small-scale structure 9. To test this possibility we have run simulations in which a predetermined area around the vortices is uniformly refined to the highest resolution. Compared to fully adaptive refinement (the default) this approach significantly reduces the amount of perturbations introduced by the grid adaption process but increases the computational cost of the simulations.

In Fig. 4 we compare the results from a fully adaptive grid and grids with maximally refined rectangles of $3 \times 3 \mathrm{~cm}, 4 \times 4 \mathrm{~cm}$, and $4 \times 8 \mathrm{~cm}$. The vortex structure is always less than $2 \mathrm{~cm}$ across. For the different grids the large scale morphology remains the same, but the shape of the cross-section visibly differs depending on the grid, as does the amount and location of small-scale structures. In particular, differences are noticeable in the small-scale instabilities present on the vortex rolls. Since all other simulation aspects are the same, the differences must originate with perturbations at jumps in refinement.

We then repeated the simulations on the different grids, but at a limiting Courant number of $\mathrm{CFL}=0.2$. The results are shown in Fig. 5 . We observe much less variation between solutions on adaptive and lo- 


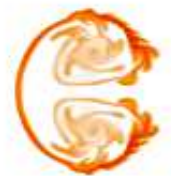

Adaptive

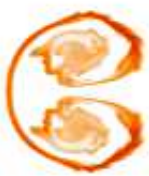

$3 \times 3$ rect

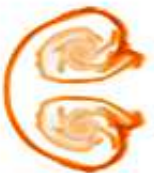

$4 \times 4$ rect

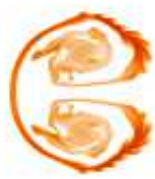

$4 \times 8$ rect

Figure 4. Solutions on different grids, $750 \mu \mathrm{s}$ after shock impact at $\mathrm{CFL}=0.8$. Left to right: fully adaptive grid; $3 \times 3 \mathrm{~cm}$ refined rectangle; $4 \times 4 \mathrm{~cm}$ refined rectangle; $4 \times 8 \mathrm{~cm}$ refined rectangle. In the rightmost image, the refined rectangle covers the entire spanwise extent of the test section.

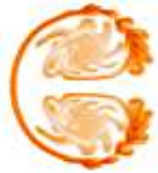

Adaptive

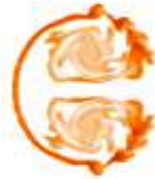

$3 \times 3$ rect

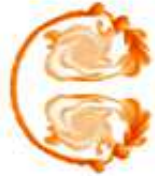

$4 \times 4$ rect

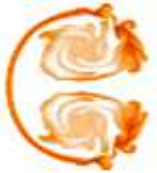

$4 \mathrm{x} 8$ rect

Figure 5. Solutions on different grids for $\mathrm{CFL}=0.2$; for other details see Fig. 4

cally uniform grids at $\mathrm{CFL}=0.2$ than at $\mathrm{CFL}=0.8$. One explanation for these results is that the errors at the fine-coarse boundaries are larger and lead to stronger perturbations at higher CFL numbers. An alternative explanation might be that at higher Courant numbers, PPM does not adequately compute solutions at these conditions. Our simulations indicate that for FLASH, a lower CFL number leads to more consistent results on different grids.

In addition to the SF6 distribution, experimental measurements of the velocity field in the vicinity of the vortex pair are available. The particle image velocimetry technique provides two-dimensional velocity vectors in the image plane. We find that in the frame of reference of the vortices, the velocity magnitude and large scale structure of our 
simulations match those measured. The greatest discrepancies are at smaller scales at the outside edges of the vortices.

Finally, we executed a speculative three-dimensional simulation. Our extension of the initial conditions in the third (cylinder axis) dimension is purely ad hoc, because we have no corresponding experimental data. For this reason this simulation cannot be used as a validation test for the FLASH code, but we hope it will open a new line of investigation and discussion. In the cylinder-axis dimension, we varied the maximum mole fraction from $\mathrm{X}_{S F 6}=0.64$ at the top wall of the test section to $\mathrm{X}_{S F 6}=0.47$ at the bottom, and in the same direction the radius of the cylinder increases slightly. These changes result in greater vorticity deposition at the top wall, and the vortex pair and instabilities evolve more quickly there. This behavior is expected based on the two-dimensional simulations; more interesting is that the maximum flow velocity in the axial direction is greater than half that in the spanwise direction by the end of the simulation $(750 \mu \mathrm{s})$. The stronger vortices have lower core pressures, and the pressure gradient in the vortex cores accelerates the flow from the bottom wall toward the top wall. The air is preferentially accelerated because of its lower molecular weight and confinement by the $\mathrm{SF}_{6}$, which acts like the wall of a tube. For the initial conditions we have assumed, the axial velocities suggest the three-dimensional effects are present. 


\section{Concluding Remarks}

To date we have made a large number of two-dimensional simulations to validate the FLASH code for problems dominated by vortex dynamics. So far, we have gained a better understanding of the sensitivity of the computed solutions to simulation parameters such as resolution, CFL number, and mesh adaption. While we can recover the overall morphology, the approximate amount and location of small scale structure, and velocity field, we must make assumptions (though reasonable) about the initial conditions to do so.

We continue to work on several fronts. We lack quantitative, physically meaningful metrics for comparing simulation and experimental data. These metrics are difficult to develop and are rarely given the attention they deserve. FLASH simulations do not currently include a

physical model for viscosity, but resolution-dependent numerical viscosity is present. Simulations with a minimum grid spacing of $78 \mu \mathrm{m}$ exhibit approximately the same amount of small scale structure as seen in the experimental data, while results on coarser grids show too little and on finer grids too much. We will soon begin simulations with models for physical diffusion; all the results presented here will then be reviewed. Our three-dimensional simulation, despite issues with the initial conditions, suggests that three-dimensional effects might be important for this experiment. We are performing a systematic study of 
three-dimensional effects, and hope that experimental data will become available for comparison.

\section{References}

1. B. Fryxell, K. Olson, P. Ricker, F. X. Timmes, M. Zingale, D. Q. Lamb, P. MacNeice, R. Rosner, J. W. Truran, and H. Tufo. FLASH: An adaptive mesh hydrodynamics code for modeling astrophysical thermonuclear flashes. ApJS, 131:273-334, November 2000.

2. A. C. Calder, B. Fryxell, T. Plewa, R. Rosner, L. J. Dursi, V. G. Weirs, T. Dupont, H. F. Robey, J. O. Kane, B. A. Remington, R. P. Drake, G. Dimonte, M. Zingale, F. X. Timmes, K. Olson, P. Ricker, P. MacNeice, and H. M. Tufo. On validating an astrophysical simulation code. ApJ Supplement Series, 143:201-229, November 2002.

3. C. Tomkins, K. Prestridge, P. Rightley, M. Marr-Lyon, P. Vorobieff, and R. Benjamin. A quantitive study of the interaction of two Richtmyer-Meshkov-unstable gas cylinders. Physics of Fluids, 15(4):986-1004, April 2003.

4. C. A. Zoldi. A Numerical and Experimental Study of a Shock-Accelerated Heavy Gas Cylinder. PhD thesis, State University of New York at Stony Brook, 2002.

5. K. Prestridge, P. Vorobieff, P. M. Rightley, and R. Benjamin. Validation of an instability growth model using particle image velocimetry measurements. Phys. Rev. Lett., 84(19):4353-4356, May 2000.

6. J. W. Jacobs. The dynamics of shock accelerated light and heavy gas cylinders. Physics of Fluids A, 5(9):2239-2247, September 1993.

7. J. J. Quirk and S. Karni. On the dynamics of a shock-bubble interaction. Journal of Fluid Mechanics, 318:129-163, 1996.

8. V. Dwarkadas, T. Plewa, G. Weirs, C. Tomkins, and M. Marr-Lyon. Simulation of vortex-dominated flows using the FLASH code. In T. Plewa, T. Linde, and V. G. Weirs, editors, Adaptive Mesh Refinement - Theory and Applications, Springer LNCSE Series. Springer-Verlag, 2004.

9. J. J. Quirk. An Adaptive Mesh Refinement Algorithm for Computational Shock Hydrodynamics. PhD thesis, Cranfield Institute of Technology, UK, 1991. 